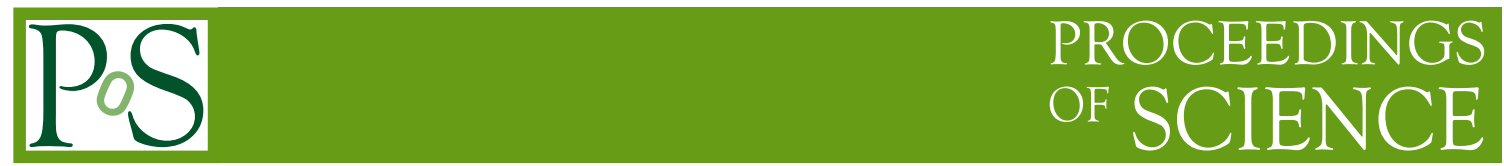

\title{
Beyond the Standard Model: Theory Status
}

\author{
Kiwoon Choi*t \\ Center for Theoretical Physics of the Universe, Institute for Basic Science \\ Daejeon, 34051, Korea \\ E-mail: kchoi@ibs.re.kr
}

I discuss the current status of theoretical efforts to explore physics beyond the Standard Model (BSM). After a short discussion of certain generic issues, I focus on the two theoretical ideas motivated by the weak scale hierarchy problem, the $\mathrm{TeV}$ scale supersymmetry which has been one of the most popular BSM physics over the past decades, and the cosmological relaxation of the Higgs boson mass, which was proposed recently as a new approach to the hierarchy problem.

38th International Conference on High Energy Physics

3-10 August 2016

Chicago, USA

\footnotetext{
* Speaker.

${ }^{\dagger}$ This work was supported by IBS under the project code IBS-R018-D1.
} 


\section{Introduction}

Standard Model (SM) of particle physics is enormously successful to explain all the available laboratory data, except the neutrino oscillations, with an impressive accuracy. Yet we have many reasons to speculate about physics beyond the SM (BSM). First of all, there are BSM physics with reliable observational evidence, e.g. neutrino oscillations, dark matter, and matter-antimatter asymmetry in the universe. There are also a variety of BSM physics which are theoretically well motivated, although not have an observational hint yet. Some of those BSM physics are introduced to understand the naturalness problems of the SM such as the weak scale hierarchy problem and the strong CP problem, or motivated by the dream for a unified theory accommodating all fundamental interactions including the quantum gravity.

So presently we have a long list of candidates for BSM physics. But the problem is that we don't know where they are. After the discovery of the Higgs boson, we don't have anymore a convincing argument to pinpoint the next scale. Indeed in most cases the scale of new physics is quite uncertain, spanning over many orders of magnitude as summarized in Fig.1. This is why we need to explore BSM physics from all possible perspectives, including the three pillars of fundamental physics together, i.e. the energy, intensity, and cosmic frontiers.

Although not convincing enough, we have two long-standing arguments which suggest that BSM physics might be near the weak scale. One is the weak scale hierarchy problem originating from the quadratically divergent radiative corrections to the Higgs boson mass, and the other is the so-called WIMP miracle which explains the amount of dark matter in a natural manner. We notice that these two arguments are still alive as many talks in the BSM session of this meeting were about the subjects related to the hierarchy problem or the WIMP dark matter.

The topics covered in the BSM session are rather broad. As mentioned above, one of the major topics is BSM physics related to the hierarchy problem, e.g. SUSY, composite Higgs, extra $\mathrm{dim}$, and relaxion. Another major topic is physics of dark sector involving dark matter and/or dark gauge bosons. We also had a special session for the $750 \mathrm{GeV}$ diphoton excess which now turns out to be a statistical fluctuation. As was reported in this meeting, the Run II LHC experiments made an impressive progress in searching for BSM physics. Unfortunately we don't have any clear sign of new physics yet, while the new data significantly improved the bounds on many popular BSM scenarios as depicted in Fig.2.

In this talk, rather than summarizing the issues discussed in the BSM session, I will focus on two specific subjects related to the hierarchy problem: the TeV scale SUSY which has been the most popular solution to the hierarchy problem over the past decades, and the cosmological relaxation which was proposed recently as a new approach to the hierarchy problem.

\section{SUSY}

Over the past several decades, the TeV scale SUSY has been the prime candidate for BSM physics near the weak scale [1]. SUSY regulates the quadratically divergent radiative corrections to the Higgs boson mass to a value comparable to the superparticle masses, suggesting that the superparticles may exist around the weak scale. The TeV scale SUSY provides also an appealing candidate for dark matter, the weakly interacting "Lightest SUSY Particle" (LSP). It gives rise to 


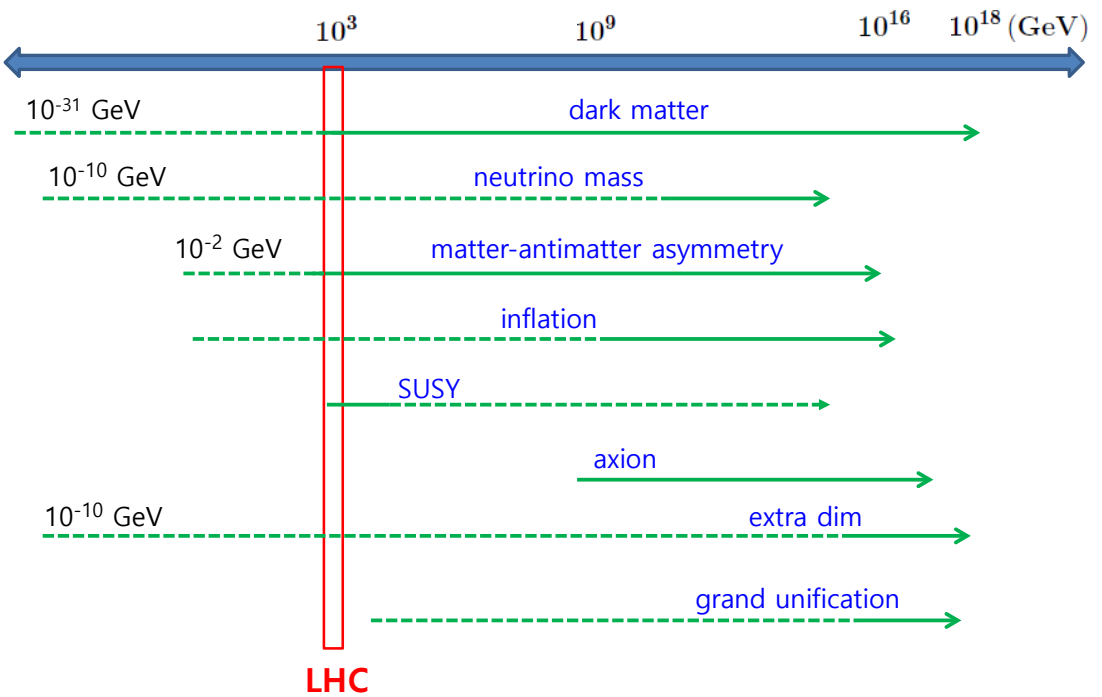

Figure 1: Possible range of scales for the popular candidates of new physics

another unexpected bonus, the successful unification of the three gauge couplings at a scale around $10^{16} \mathrm{GeV}$, and has natural connection to string theory.

It has been regarded for long time that the naturalness argument on the Higgs boson mass provides a major hint on the SUSY scale. In SUSY models, the up-type Higgs boson mass at the weak scale includes the piece

$$
\Delta m_{H_{u}}^{2}=\mu^{2}-\frac{3 y_{t}^{2}}{4 \pi^{2}}\left(m_{\tilde{t}}^{2}+\frac{g_{s}^{2}}{3 \pi^{2}} m_{\tilde{g}}^{2} \ln \left(\frac{\Lambda_{m}}{m_{\tilde{g}}}\right)\right) \ln \left(\frac{\Lambda_{m}}{m_{\tilde{t}}}\right),
$$

where $\mu$ is the Higgsino mass, $y_{t}$ is the top quark Yukawa couplings, and the piece depending on $\ln \Lambda_{m}$ represents the radiative corrections from the loops involving the stops $\tilde{t}$ and the gluinos $\tilde{g}$. The mediation scale $\Lambda_{m}$ corresponds to the scale where the soft SUSY breaking terms are generated as effective local operators, which can be anywhere between the Planck scale and the superparticle masses, depending upon how SUSY breaking in the hidden sector is transmitted to the visible sector. The naturalness argument tells that each piece of $\Delta m_{H_{u}}^{2}$ should not significantly exceed the weak scale in order to avoid a fine tuning problem, implying that the Higgsinos, stops, and gluinos may not be far away from the weak scale.

In fact, the above argument is not convincing enough as a small Higgs mass may be achieved by the anthropic selection, or by the recently proposed relaxation mechanism. Yet there are other hints indicating that SUSY may not be too far away from the weak scale. In SUSY models, the mass of the SM-like Higgs boson is given by

$$
m_{h}^{2}=M_{Z}^{2} \cos ^{2} 2 \beta+\frac{3 y_{t}^{2} m_{t}^{2}}{4 \pi^{2}} \ln \left(\frac{m_{\tilde{t}}}{m_{t}}\right)+\ldots
$$

where the ellipsis stands for the subleading part. Combined with the measured Higgs boson mass, $m_{h} \simeq 125 \mathrm{GeV}$, the above relation indicates that the stop masses, or generic sfermion masses, are below $10^{3} \mathrm{TeV}$ for $\tan \beta>2$. If one takes the successful gauge coupling unification seriously, one finds that the Higgino mass is bounded to be below about $10 \mathrm{TeV}$. These hints on the SUSY scale 

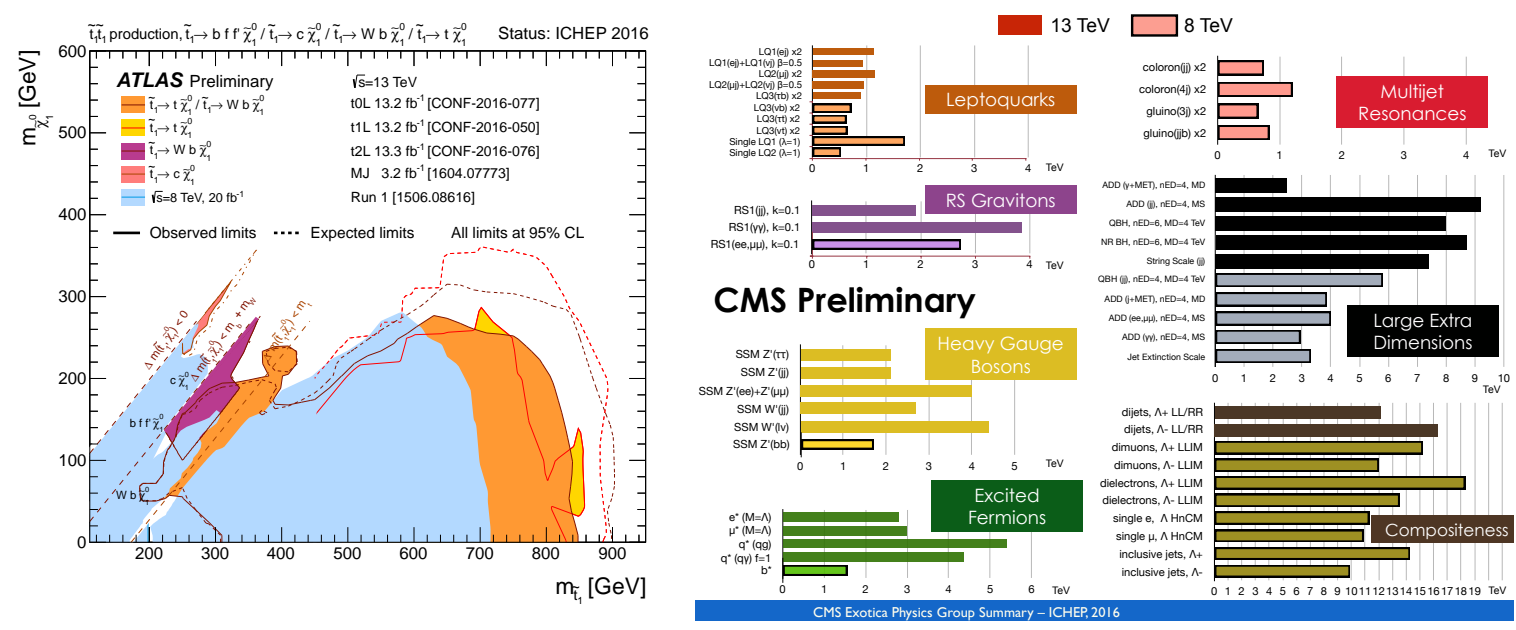

Figure 2: ATLAS exclusion limit on the stop mass and CMS bounds on BSM physics other than SUSY

never guarantee that SUSY is within the reach of the LHC experiments, but leave us a room to hope that SUSY may be just around the corner.

There can be a variety of different SUSY signatures at the LHC, which were extensively discussed in this meeting. Some of the best studied examples include multi-jets (possibly with additional leptons or photons) with missing transverse momentum, displaced vertices, and long-lived particle tracks. Detailed feature of SUSY signatures depends on the underlying SUSY model, particularly on i) the nature of LSP, ii) the shape of SUSY spectrum (compressed or split), and iii) the validity of the $R$-parity conservation.

Theoretically the SUSY spectrum and the nature of LSP are determined mostly by the mediation mechanism of SUSY breaking. Typical models for SUSY breaking involve a hidden sector which breaks SUSY spontaneously through the vacuum values of the auxiliary components of some chiral superfields $X$, vector superfields $V_{A}$, and the chiral compensator $C$ in the supergravity multiplet:

$$
\langle X\rangle=X_{0}+\theta^{2} F^{X}, \quad\left\langle V_{A}\right\rangle=\frac{1}{2} \theta^{2} \bar{\theta}^{2} D_{A}, \quad\langle C\rangle=C_{0}+\theta^{2} F^{c},
$$

where the compensator $F$-component is determined as $F^{C} / C_{0}=m_{3 / 2}+\frac{1}{3} F^{X} \partial_{X} K_{0}$ for the Kähler potential $K_{0}$ of $X$. At the messenger scale $\Lambda_{m}$, the effects of SUSY breaking can be encoded in the following form of effective interactions between the SUSY breaking fields and the visible sector fields [2]:

$$
\begin{aligned}
\mathscr{L}_{\text {edd }} & =\int d^{2} \theta d^{2} \bar{\theta} C C^{*} Y_{I}\left(Z, Z^{*}, V_{A}\right) \Phi_{I}^{*} \Phi_{I} \\
& +\left(\int d^{2} \theta\left(\frac{1}{4} f_{a}(Z) \mathscr{W}^{a \alpha} \mathscr{W}_{\alpha}^{a}+C^{3} \frac{\lambda_{I J K}(X)}{6} \Phi_{I} \Phi_{J} \Phi_{K}\right)+\text { c.c }\right),
\end{aligned}
$$

where $Z=(X, C)$, and $\mathscr{W}_{\alpha}^{a}$ and $\Phi_{I}$ stand for the visible gauge and matter multiplets, respectively. One then finds the soft SUSY breaking terms of the canonically normalized scalar fields and gaug- 
inos in the visible sector, which are renormalized at $\Lambda_{m}$, are given by

$$
\mathscr{L}_{\text {soft }}=-m_{I}^{2}\left|\phi^{I}\right|^{2}-\frac{1}{2}\left(M_{a} \lambda^{a} \lambda^{a}+\frac{1}{6} A_{I J K} y_{I J K} \phi^{I} \phi^{J} \phi^{K}+\text { c.c }\right),
$$

where $y_{I J K}=\lambda_{I J K} / \sqrt{Y_{I} Y_{J} Y_{K}}$ denote the physical Yukawa couplings, and

$$
\begin{aligned}
m_{I}^{2}\left(\Lambda_{m}\right) & =-F^{Z} F^{\bar{Z}} \partial_{Z} \partial_{\bar{Z}} \ln Y_{I}-\frac{1}{2} D_{A} \frac{\partial}{\partial V_{A}} \ln Y_{I} \\
& =-\frac{1}{4} \frac{d \gamma_{I}}{d \ln p}\left|\frac{F^{C}}{C_{0}}\right|^{2}-\frac{1}{2}\left(\frac{F^{C}}{C_{0}} F^{\bar{X}} \partial_{\bar{X}}+\frac{F^{C *}}{C_{0}^{*}} F^{X} \partial_{X}\right) \gamma_{I}-F^{X} F^{\bar{X}} \partial_{X} \partial_{\bar{X}} \ln Y_{I}-\frac{1}{2} D_{A} \frac{\partial}{\partial V_{A}} \ln Y_{I}, \\
\frac{M_{a}(p)}{g_{a}^{2}(p)} & =\left.F^{Z} \partial_{Z} \mathscr{F}_{a}\right|_{C_{0}=e^{K_{0} / 6}} \\
& =\frac{\frac{1}{16 \pi^{2}}\left(b_{a}+\sum_{I} \operatorname{tr}\left(T_{a}^{2}\left(\Phi_{I}\right) \gamma_{I}\right) \frac{F^{C}}{C_{0}}+F^{X} \partial_{X}\left(\operatorname{Re} f_{a}\right)-\frac{1}{8 \pi^{2}} \sum_{I} \operatorname{tr}\left(T_{a}^{2}\left(\Phi_{I}\right)\right) F^{i} \partial_{i} \ln \left(Y_{I}\right)\right.}{1-\frac{1}{8 \pi^{2}} \operatorname{tr}\left(T_{a}^{2}(\operatorname{adj})\right) g_{a}^{2}} \\
A_{I J K}\left(\Lambda_{m}\right) & =-F^{Z} \partial_{Z} \ln \left(\frac{\lambda_{I J K}}{Y_{I} Y_{J} Y_{K}}\right)=-\frac{1}{2}\left(\gamma_{I}+\gamma_{J}+\gamma_{K}\right) \frac{F^{C}}{C_{0}}-F^{X} \partial_{X} \ln \left(\frac{\lambda_{I J K}}{Y_{I} Y_{J} Y_{K}}\right),
\end{aligned}
$$

for the 1PI gauge coupling superfield [2]:

$$
\begin{aligned}
\mathscr{F}_{a}\left(p^{2}\right) & =\left.\operatorname{Re}\left(f_{a}\right)\right|_{C=1}+\frac{b_{a}}{16 \pi^{2}} \ln \left(\frac{C C^{*} \Lambda_{m}^{2}}{p^{2}}\right)-\frac{1}{8 \pi^{2}} \sum_{I} \operatorname{tr}\left(T_{a}^{2}\left(\Phi_{I}\right)\right) \ln \left(Y_{I}\left(p^{2} / C C^{*}\right)\right) \\
& +\frac{1}{8 \pi^{2}} \operatorname{tr}\left(T_{a}^{2}(\operatorname{adj})\right) \ln \mathscr{F}_{a}\left(p^{2} / C C^{*}\right)
\end{aligned}
$$

and the anomalous dimension of the matter fields: $\gamma_{I}=d \ln Y_{I} / d \ln p$.

There are three popular mediation schemes of SUSY breaking, (i) gravity (or moduli) mediation due to $X$ which couples to the visible sector with gravitational strength [3], i.e. suppressed by $1 / M_{\text {Planck }}$, (ii) gauge mediation due to $X$ which has a renormalizable Yukawa coupling to massive gauge charged messenger fields [4], yielding $X$-dependent thresholds corrections to $f_{a}$ and $Y_{I}$ after the messenger fields are integrated out, and (iii) anomaly mediation due to the chiral compensator $C$ [5]. The $D$-term of hidden sector vector superfield can be also an important source of scalar masses. It is in fact a plausible possibility, particularly in view of the known scenarios of string moduli stabilization, that some or all of these mediations give comparable contributions to SUSY breaking masses, yielding a variety forms of mixed mediations [6]. Such mixed mediations make the theory space for superparticle spectrum big and rich, leading to a variety of different patterns of SUSY spectrum, including the split or compressed SUSY scenario.

As SUSY has been the prime candidate for BSM physics over the past several decades, we are now ready well to interpret any potential SUSY signature at the LHC. The simplest interpretation for the absence of SUSY signature would be that SUSY is too heavy to be discovered in the presently available data. There are however other possibilities, which should be extensively explored in the forthcoming LHC experiments. A particularly interesting possibility is the so-called stealth SUSY scenario [8] involving a stealth sector with relatively suppressed SUSY breaking, together with a light gravitino or axino LSP. In such scenario, SUSY events have soft missing energy, which would allow colored SUSY particles to be in sub-TeV region [8]. Another interesting 
possibility is that EW SUSY is in the range of $\mathscr{O}(100) \mathrm{GeV}$, which may explain the $3.5 \sigma$ deviation of the anomalous magnetic moment of the muon, while colored SUSY is in multi-TeV region. This possibility of light EW SUSY must be one of the key target of the high luminosity LHC experiment and also of the ILC experiment.

Even when the SUSY scale is well above the weak scale, and therefore out of reach from collider experiments in foreseeable future, SUSY may leave an imprint in the low energy precision data. It has been noted that low energy meson mixing can probe the squark or slepton masses up to $\mathscr{O}\left(10^{3}\right) \mathrm{TeV}$ [9]. Even when sfermions are heavier than $\mathscr{O}\left(10^{3}\right) \mathrm{TeV}$, if the EW gauginos and Higgsinos are below $\mathscr{O}(10) \mathrm{TeV}$ as suggested by the gauge coupling unification, generically the resulting nucleon EDMs $d_{N} \gtrsim \mathscr{O}\left(10^{-30}\right) e \cdot \mathrm{cm}[10]$, which might be probed by the proposed storage ring EDM experiment [11].

\section{Relaxion}

Recently cosmological relaxation of the Higgs boson mass has been proposed as an alternative solution to the weak scale hierarchy problem [12]. In this scenario, a pseudo Nambu-Goldston boson $\phi$ is coupled to the Standard Model Higgs doublet $H$, scanning the Higgs mass parameter $m_{H}^{2}$ from an initial value of $\mathscr{O}\left(\Lambda^{2}\right)$ to the present value $m_{H}^{2}=-(89 \mathrm{GeV})^{2}$, where $\Lambda$ is the Higgs mass cutoff presumed to be much larger than the weak scale $v=246 \mathrm{GeV}$. This scalar field, often referred as relaxion, has a potential including the piece enforcing the relaxion to move to scan the Higgs mass, and also a periodic barrier potential to stop the relaxion at the position giving $m_{H}^{2}=-(89 \mathrm{GeV})^{2}$. More explicitly, the scalar potential is given by

$$
-\left(\Lambda^{2}-\frac{\Lambda^{2}}{f_{\mathrm{eff}}} \phi\right)|H|^{2}-c_{0} \frac{\Lambda^{4}}{f_{\mathrm{eff}}} \phi+V_{b}
$$

where $f_{\text {eff }}$ corresponds to the relaxion excursion required to scan $m_{H}^{2}$ from $\mathscr{O}\left(\Lambda^{2}\right)$ to its final value, $c_{0}$ is a positive coefficient bounded as $c_{0} \gtrsim \mathscr{O}\left(1 / 16 \pi^{2}\right)$ by the naturalness argument, and the barrier potential $V_{b}$ takes the form:

$$
V_{b}=-\Lambda_{b}^{4}(H) \cos (\phi / f)
$$

with a Higgs-dependent amplitude $\Lambda_{b}^{4}(H)=\mu_{b}^{4-n} H^{n}$, where $\mu_{b}$ is determined by the scale where $V_{b}$ is generated. Imposing the stationary condition, one finds

$$
\frac{f_{\text {eff }}}{f} \sim \frac{\Lambda^{4}}{\Lambda_{b}^{4}(H=v)} \frac{c_{0}}{\sin \left(\phi_{0} / f\right)},
$$

where $\phi_{0}$ denotes the relaxion vacuum value in the present universe.

There are two ways to generate the barrier potential, which have been discussed so far in the literatures $[12,13,14]$. The minimal scenario is to generate $V_{b}$ through the relaxion coupling $\phi G \tilde{G} / 32 \pi^{2} f$, where $G$ and $\tilde{G}$ denote the gluon field strength and its dual, which would result in

$$
\Lambda_{b}^{4}(H) \sim y_{u} H \Lambda_{\mathrm{QCD}}^{3},
$$

where $\Lambda_{\mathrm{QCD}} \sim 200 \mathrm{MeV}$ is the QCD scale and $y_{u} \sim 10^{-5}$ is the up-quark Yukawa coupling. In this case, $\phi_{0} / f$ is identified as the QCD vacuum angle $\theta_{\mathrm{QCD}}$ and therefore constrained as $\left|\sin \left(\phi_{0} / f\right)\right| \lesssim$ 


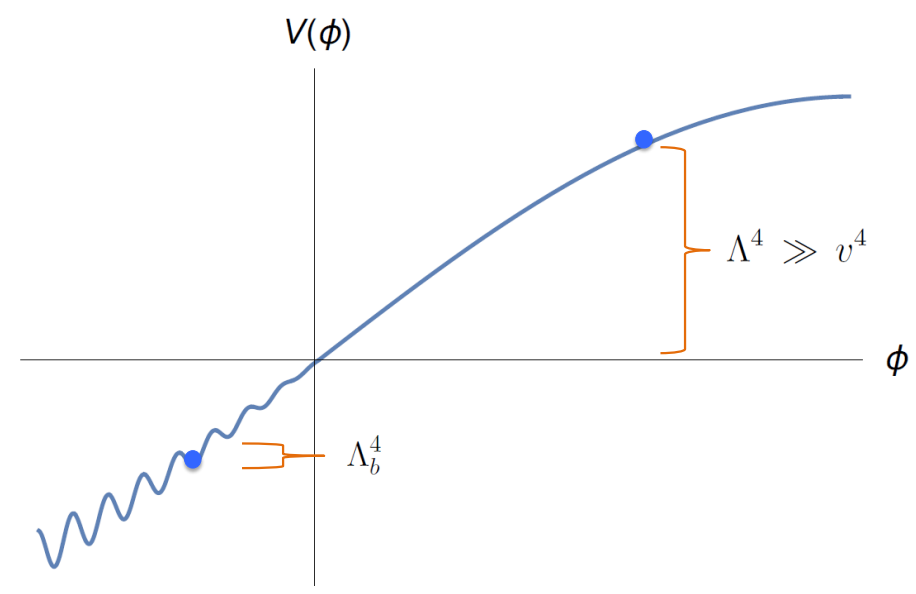

Figure 3: Typical relaxion potential, where the bullets represent the initial and final relaxion position.

$10^{-9}$. Alternatively, the barrier potential can be generated by a new physics around the weak scale, yielding for instance

$$
\Lambda_{b}^{4}(H)=\mu_{b}^{2}|H|^{2}
$$

with $\mu_{b} \lesssim \mathscr{O}(4 \pi v)$ and $\sin \left(\phi_{0} / f\right)=\mathscr{O}(1)[13,14]$. In Fig. (3), we depict the typical shape of the relaxion potential, including the periodic $V_{b}$.

A key ingredient of the relaxion scenario is a mechanism to dissipate away the relaxion kinetic energy originating from the initial potential energy of $\mathscr{O}\left(\Lambda^{4}\right)$. It is usually assumed that the relaxion loses its kinetic energy by the Hubble friction during the inflationary period ${ }^{1}$. Then the scheme requires a rather large number of inflationary e-foldings, e.g.

$$
N_{e} \sim \frac{\Lambda^{4}}{\Lambda_{b}^{4}} \gtrsim\left(\frac{\Lambda}{\mathrm{TeV}}\right)^{4}
$$

for the case that the barrier potential is induced by new physics, and

$$
N_{e} \sim \frac{\Lambda^{4}}{\theta_{\mathrm{QCD}} y_{u} v \Lambda_{\mathrm{QCD}}^{3}} \gtrsim 10^{24}\left(\frac{\Lambda}{\mathrm{TeV}}\right)^{4}
$$

for the other case that the barrier potential is induced by low energy QCD. The above result and the relaxion scale hierarchy (3.2) show that the scenario with QCD-induced barrier potential requires a huge e-folding number and also a big hierarchy among the relaxion scales, which might be difficult to be achieved without causing a fine-tuning problem.

The stationary condition (3.2) shows that the relaxion mechanism transmutes the weak scale hierarchy $\Lambda \gg v$ to another hierarchy $f_{\text {eff }} \gg f$. Although the latter hierarchy can be technically natural, it may require an explanation for its origin. This issue has been addressed in $[16,17]$, proposing a scheme to generate an exponential hierarchy $f_{\text {eff }} / f \sim e^{N}$ based on models with $N$

\footnotetext{
${ }^{1}$ See [15] for another possibility to dissipate the relaxion energy through gauge field production.
} 
axions [18]. To illustrate the basic idea, one can consider a model with $N$ axions with the following lagrangian density:

$$
\mathscr{L}=\frac{1}{2} \sum_{i}\left(\partial_{\mu} \phi_{i}\right)^{2}-\left(\tilde{V}_{0}+V_{0}+\mu_{h}^{2}|H|^{2}+V_{b}+\ldots\right),
$$

where

$$
\begin{aligned}
& \tilde{V}_{0}=-\sum_{i=1}^{N-1} M_{i}^{4} \cos \left(\frac{\phi_{i}}{f_{i}}+n_{i} \frac{\phi_{i+1}}{f_{i+1}}\right), \quad V_{0}=-\Lambda_{0}^{4} \cos \left(\frac{\phi_{N}}{f_{N}}+\delta_{N}\right), \\
& \mu_{h}^{2}=\Lambda_{1}^{2}-\Lambda_{2}^{2} \cos \left(\frac{\phi_{N}}{f_{N}}\right), \quad V_{b}=-\Lambda_{b}^{4}(H) \cos \left(\frac{\phi_{1}}{f_{1}}+\delta_{1}\right),
\end{aligned}
$$

with $M_{i} \gg \Lambda_{0} \sim \Lambda_{1} \sim \Lambda_{2} \gg \Lambda_{b}$ and all $f_{i}$ are comparable to each other. The dominant part of the potential, i.e. $\tilde{V}_{0}$, provides the so-called clockwork gear whose flat direction is identified [16] as

$$
\phi \propto \sum_{i=1}^{N}(-1)^{i-1}\left(\prod_{j=i}^{N-1} n_{j}\right) f_{i} \phi_{i} .
$$

From $\tilde{V}_{0}, N-1$ axions get heavy masses and can be integrated out, leaving a low energy effective theory of the light axion $\phi$. One then finds the original $N$ axions can be parametrized in terms of the canonically normalized $\phi$ as

$$
\frac{\phi_{i}}{f_{i}}=(-1)^{i-1}\left(\prod_{j=i}^{N-1} n_{j}\right) \frac{\phi}{f_{\mathrm{eff}}}
$$

where

$$
f_{\text {eff }}=\sqrt{\sum_{i=1}^{N}\left(\prod_{j=i}^{N-1} n_{j}^{2}\right) f_{i}^{2}} \sim\left(\prod_{j=1}^{N-1} n_{j}\right) f_{1} .
$$

The resulting effective potential of $\phi$ involves two hierarchical axions scales $f$ and $f_{\mathrm{eff}}[16,17]$ :

$$
V_{\mathrm{eff}}=-\Lambda_{0}^{4} \cos \left(\frac{\phi}{f_{\mathrm{eff}}}+(-)^{N-1} \delta_{N}\right)+\left(\Lambda_{1}^{2}-\Lambda_{2}^{2} \cos \left(\frac{\phi}{f_{\mathrm{eff}}}\right)\right)|h|^{2}-\Lambda_{b}^{4}(h) \cos \left(\frac{\phi}{f}+\delta_{1}\right),
$$

where

$$
\frac{f_{\mathrm{eff}}}{f}=\left(\prod_{j=1}^{N-1} n_{j}\right)=\mathscr{O}\left(e^{N}\right) .
$$

Usually the dynamics generating a periodic barrier potential induces also the relaxion-photon coupling

$$
\frac{c_{\phi \gamma}}{32 \pi^{2}} \frac{\phi}{f} F^{\mu v} \tilde{F}_{\mu v}
$$

where $c_{\phi \gamma}$ is a model-dependent coefficient, being generically of order unity. In addition to this, the barrier potential with the amplitude (3.4) yields a relaxion-Higgs mass mixing, which results in 


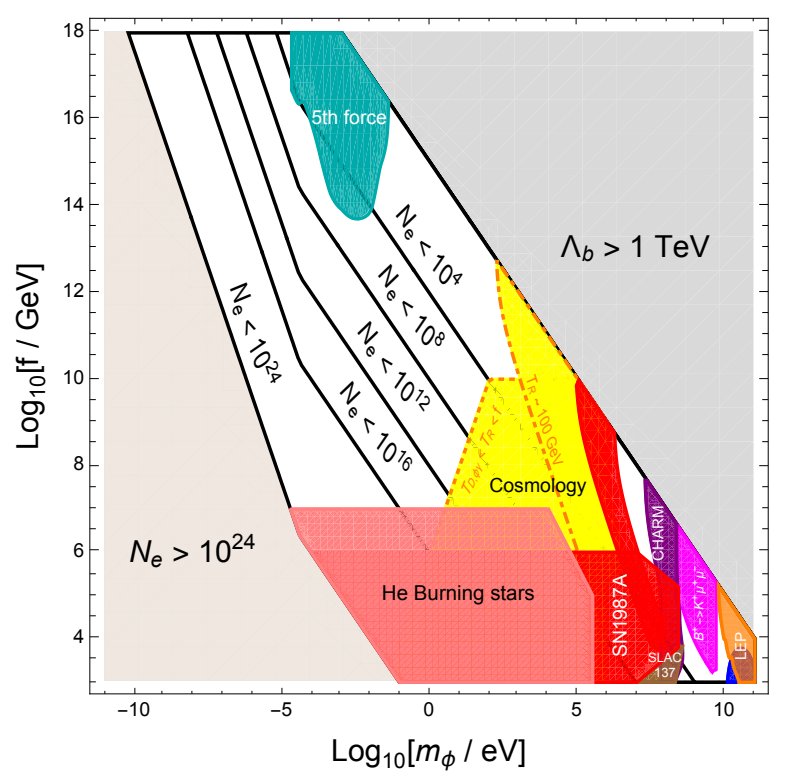

Figure 4: Cosmological relaxion window with colored regions excluded by the observational constraints.

various forms of low energy couplings between the relaxion and the SM fields [19]. Applying those relaxion couplings to various low energy processes, one can derive the observational constraints on the relaxion mass $m_{\phi}$ and the decay constant $f$ [19]. The results is summarized in Fig. (4) over the parameter region with $N_{e}<10^{24}$ and $\Lambda_{b}<1 \mathrm{TeV}$. Here the colored regions are excluded by the constraints from the LEP data, EDM, rare meson decays, beam dump experiments, astrophysical and cosmological considerations, and the 5 th force. The yellow region is from relaxion cosmology depending on the reheating temperature, and shrinks for lower reheating temperature.

One can see that essentially there are three distinct viable windows on the relaxion parameter space: i) a window with $f \sim$ few $-200 \mathrm{TeV}$ and $m_{\phi} \sim 0.2-10 \mathrm{GeV}$, ii) another window with $f \sim 10^{6}-10^{9} \mathrm{GeV}$ and $m_{\phi} \sim$ few $-50 \mathrm{MeV}$, and finally iii) the biggest window with $f>10^{7} \mathrm{GeV}$ and $m_{\phi} \lesssim 100 \mathrm{eV}$. Among these three windows, the first one is particularly interesting as it is within the reach of near future experiments. For the region with $m_{\phi} \lesssim 3 \mathrm{GeV}$ on this window, relaxions decay dominantly into photons, and also into muons or pions with comparable branching ratio, which would allow this region probed by the SHiP experiment [20]. This region can be probed also by the future storage ring EDM experiment [11] which is claimed to improve the present bounds on the nucleon EDMs by several orders of magnitudes.

There are several more issues which need to be examined further. One of them is the coincidence problem arising from the fact that the scale $\Lambda_{\mathrm{NP}}$ where the barrier potential is generated should be around the weak scale. One possible solution to this problem has been proposed in [21], introducing another relaxion-like field scanning $\Lambda_{b}^{4}$, which would allow $\Lambda_{\mathrm{NP}}$ comparable to the Higgs mass cutoff scale $\Lambda$. Another issue is the large number of inflationary e-foldings required in the limit $\Lambda \gg v$ as shown in (3.6). An interesting possibility to avoid this problem is to slow down the relaxion motion by gauge field production, rather than by the Hubble friction during the inflationary period, which was proposed recently in [15]. Of course, one eventually needs a sensible UV completion of the whole scheme, including the schemes to generate the primordial density 
perturbation, matter-antimatter asymmetry, and correct amount of dark matter in the early universe.

\section{Conclusion}

There are many motivations for BSM physics, including those based on reliable observational evidence. On the other hand, the scale of BSM physics is quite uncertain, usually spanning over many orders of magnitudes, and this makes the search for BSM physics unusually difficult. Yet the weak scale hierarchy problem and the WIMP miracle provide the major driving force for our search for BSM physics near the weak scale, and hopefully SUSY or WIMP dark matter might be just around the corner. The recently proposed cosmological relaxation of the Higgs mass is a new approach to the hierarchy problem, which certainly deserves further attention. At any rate, to make a further progress we will need a guide from experiments. In regard to this, together with the LHC Run II experiments, the precision physics frontier is expected to play a key role in our search for BSM physics.

\section{References}

[1] H. P. Nilles, Phys. Rept. 110 (1984) 1; H. E. Haber and G. L. Kane, Phys. Rept. 117 (1985)

[2] V. S. Kaplunovsky and J. Louis, Phys. Lett. B 306, 269 (1993); A. Brignole, L. E. Ibanez and C. Munoz, Nucl. Phys. B 422 (1994) 125; N. Arkani-Hamed, G. F. Giudice, M. A. Luty and R. Rattazzi, Phys. Rev. D 58, 115005 (1998).

[3] H. P. Nilles, Phys. Lett. B 115 (1982); A. H. Chamseddine, R. Arnowitt and P. Nath, Phys. Rev. Lett. 49 (1982) 970; R. Barbieri, S. Ferrara and C. A. Savoy, Phys. Lett. B 119 (1982) 343.

[4] M. Dine, W. Fischler and M. Srednicki, Nucl. Phys. B 189, 575 (1981); S. Dimopoulos and S. Raby, Nucl. Phys. B 192, 353 (1981); M. Dine and A. E. Nelson, Phys. Rev. D48, 1277 (1993).

[5] L. Randall and R. Sundrum, Nucl. Phys. B 557, 79 (1999); G. F. Giudice, M. A. Luty, H. Murayama and R. Rattazzi, JHEP 9812, 027 (1998).

[6] K. Choi, A. Falkowski, H. P. Nilles and M. Olechowski, Nucl. Phys. B 718, 113 (2005); K. Choi, K. S. Jeong and K. i. Okumura, JHEP 0509, 039 (2005); L. L. Everett, I. W. Kim, P. Ouyang and K. M. Zurek, Phys. Rev. Lett. 101, 101803 (2008);

[7] N. Arkani-Hamed and S. Dimopoulos, JHEP 0506, 073 (2005).

[8] J. Fan, M. Reece and J. T. Ruderman, JHEP 1111 (2011) 012; J. Fan, R. Krall, D. Pinner, M. Reece and J. T. Ruderman, JHEP 1607, 016 (2016).

[9] W. Altmannshofer, R. Harnik and J. Zupan, JHEP 1311, 202 (2013).

[10] G. F. Giudice and A. Romanino, Phys. Lett. B 634, 307 (2006).

[11] Y. K. Semertzidis, EPJ Web Conf. 118, 01032 (2016).

[12] P. W. Graham, D. E. Kaplan and S. Rajendran, Phys. Rev. Lett. 115, no. 22, 221801 (2015).

[13] O. Antipin and M. Redi, JHEP 1512, 031 (2015).

[14] R. S. Gupta, Z. Komargodski, G. Perez and L. Ubaldi, JHEP 1602, 166 (2016).

[15] A. Hook and G. Marques-Tavares, arXiv:1607.01786 [hep-ph]. 
[16] K. Choi and S. H. Im, JHEP 1601, 149 (2016)

[17] D. E. Kaplan and R. Rattazzi, Phys. Rev. D 93, no. 8, 085007 (2016).

[18] J. E. Kim, H. P. Nilles and M. Peloso, JCAP 0501 (2005) 005; K. Choi, H. Kim and S. Yun, Phys. Rev. D 90, 023545 (2014).

[19] K. Choi and S. H. Im, arXiv:1610.00680 [hep-ph]; T. Flacke, C. Frugiuele, E. Fuchs, R. S. Gupta and G. Perez, arXiv:1610.02025 [hep-ph].

[20] S. Alekhin et al., arXiv:1504.04855 [hep-ph].

[21] J. R. Espinosa, C. Grojean, G. Panico, A. Pomarol, O. PujolÃăs and G. Servant, Phys. Rev. Lett. 115, no. 25,251803 (2015). 